\title{
Kommerzieller Bananenanbau in Zentralamerika
}

Im Juli und August 1972 hatte der Verfasser Gelegenheit, in verschiedenen Produktionsgebieten Zentralamerikas die Wandlungen zu untersuchen, die in den letzten zwanzig Jahren den kommerziellen Anbau von Bananen mehr als irgend einen andern Sektor der dortigen Agrarproduktion umgestaltet hatten.(1) Dankbar sei an dieser Stelle die Unterstützung verdankt, welche die United Fruit Company durch Dokumentation (2) und Führung in den Anbaugebieten selbst gewährte.

Versucht man in die vielfältig miteinander verknüpften Wandlungen zum besseren Verständnis eine gewisse Ordnung zu bringen, dann beginnt man wohl am besten bei der Sortenwahl. Von den vielen Arten der Banane (musa sapiens) wurde früher vor allem die in West-Indien gezüchtete Varietät Gros-Michel angepflanzt. Diese hatte den grossen Nachteil, dass sie besonders anfällig auf die sogenannte Panamakrankheit (fusarium oxysporum f. cubense) war。 Neupflanzungen mussten oft schon nach fünf Jahren wieder aufgegeben werden, selten überdauerten sie fünfzehn Jahre。 Dies machte den Bananenanbau zu einem Produktionssektor mit hohem Landverschleiss, zwang zur Sicherstellung grosser Landreserven und wurde ganz allgemein wirtschaftlich mit der Zeit untragbar。

Das Heranzüchten einer neuen Varietät ist bei Bananen ein besonders langwieriger Prozess. Der Versuchsanstalt der United Fruit Company (UF) in Honduras gelang es, eine gegen die Panamakrankheit resistente Banane heranzuzüchten, die heute allgemein angebaut wird. Die näheren Details wurden nicht bekannt gegeben (3). Der Name der neuen Varietät lautet Valery. Auf diese Weise wurde eine weitgehende Stabilisierung der Produktionsareale erreicht, was wohl im Hinblicke auf die politische Belastung durch ungenutzte Landreserven und die beträchtlichen Investierungen in der Infrastruktur am eintscheidendsten ist. Eine weitere Krankheit, Sigatoka (Mycosphaerella musicola), welche die Blätter befällt, wurde früher über ein weit verzweigtes Röhrensystem arbeitsintensiv durch Bespritzen vom Boden aus mit Bordeauxbrühe erfolgreich aber teuer bekämpft。 Bananenpflanzungen erhielten durch das Bespritzen mit Bordeauxbrühe eine charakteristische Blaufärbung. Heute bekämpft man Sigatoka vom Flugzeuge aus. Verspritzt wird eine Mischung von Texaco 522 und Dithane M 22, eine gelbliche Brühe. Man kann nur am frühen Morgen bei Temperaturen unter $28-30^{\circ} \mathrm{C}$ spritzen, das heisst, dass sich die Zahl der täglich möglichen Flüge auf 3 bis 4 reduziert。 Je Flug können rund 150 ha bespritzt werden; das Auffüllen des Flugzeuges dauert nur elf Sekunden. Das neue Verfahren soll nur etwa einen Drittel des früheren kosten。Die Blaufärbung der Pflanzung ist verschwunden。Das gleiche Flugzeug kann später im Tag dazu verwendet werden, in Wasser gelöste Stickstoffdünger zu applizieren.

Freilich spielen auch heute noch andere Erkrankungen der Blätter, der Früchte und der Wurzelwerke eine wichtige Rolle。 Letzteres wird von einer Art Würmern (Nematoden) (4) befallen, denen an sich ebenso schwer beizukommen ist wie der Panamakrankheit。 Der - Schaden ist in diesem Falle bedeutend geringer. Valery unterscheidet sich von Gros-Michel auch morphologisch. Die Pflanze besitzt unter anderem eine gedrungenere Gestalt und es kann enger gepflanzt werden. In Verbindung mit anderen Eigenschaften drückt sich dies in einer Zunahme der Erträge je Flächeneinheit aus. So finden wir beispielsweise (5) für Honduras folgende Landesmittel: (in $\mathrm{kg} / \mathrm{ha}$ ) $1948-52=140,1961-65=142,1967=184$, $1968=190,1969=197,1970=215$ 。

Die Anlage der Pflanzungen selbst hat sich wenig verändert. Dagegen finden sich eine Reihe von Verbesserungen bis zum Zeitpunkte der Ernte. Wenn sich die Fruchtstände schon kräftig entwickelt haben, werden sie mit Plastiksäcken überzogen (bagging) und diese auf vielen Farmen mit farbigen Aufklebbändern markiert, was die Selektion im Zeitpunkte der Ernte erleichtert。 Regelmässig werden in der Folge die Fruchtstände desinfiziert, wobei vom Boden aus das Mittel von unten in den Plastiksack gespritzt wird (spraying). Gleichzeitig sichert man die Bananenstauden durch Spannen von starken Nylonschnüren gegen Windschäden, was ebenfalls neu ist und sich gut bewährt haben soll。Von Details abgeseher ist der Erntevorgang derselbe wie früher: Der cutter sticht den Stamm an, der backer empfängt die niederkommenden Fruchtstände auf seiner Schulter und bringt sie zur Stelle, von wo der Abtransport beginnt. Früher wurden die Fruchtstände (bunches, racimos) auf Wagen, Maultieren usw. zur nächsten Eisenbahnverladestelle gebracht, dort durch Eintauchen in Tanks von der Bordeauxbrühe befreit, und ohne jede weitere Veränderung verladen, im 


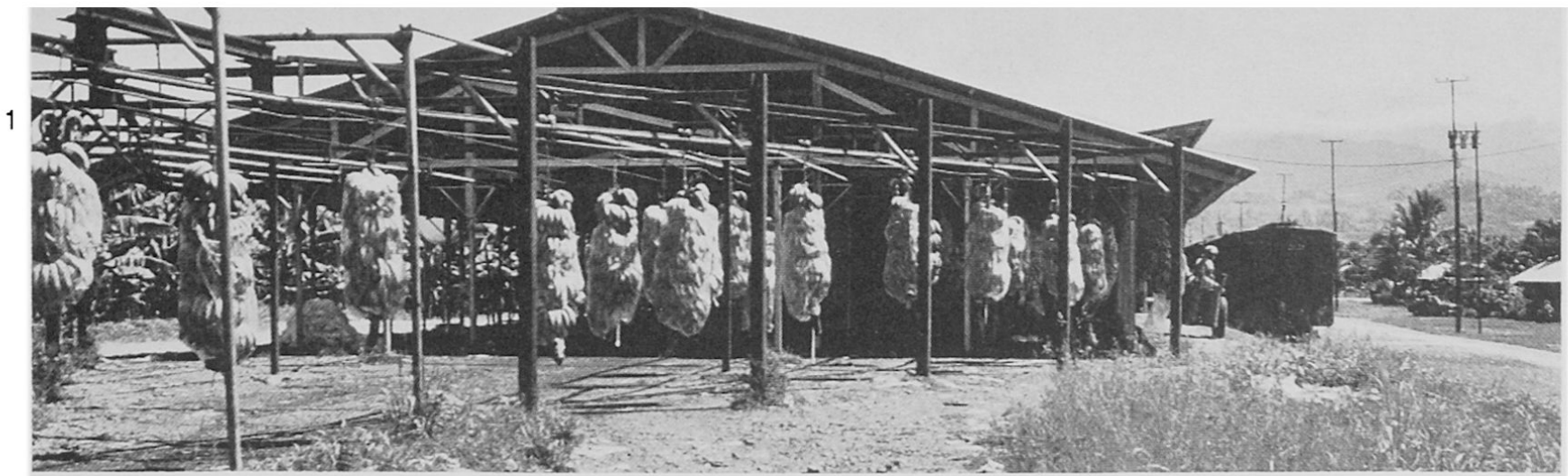

2 -

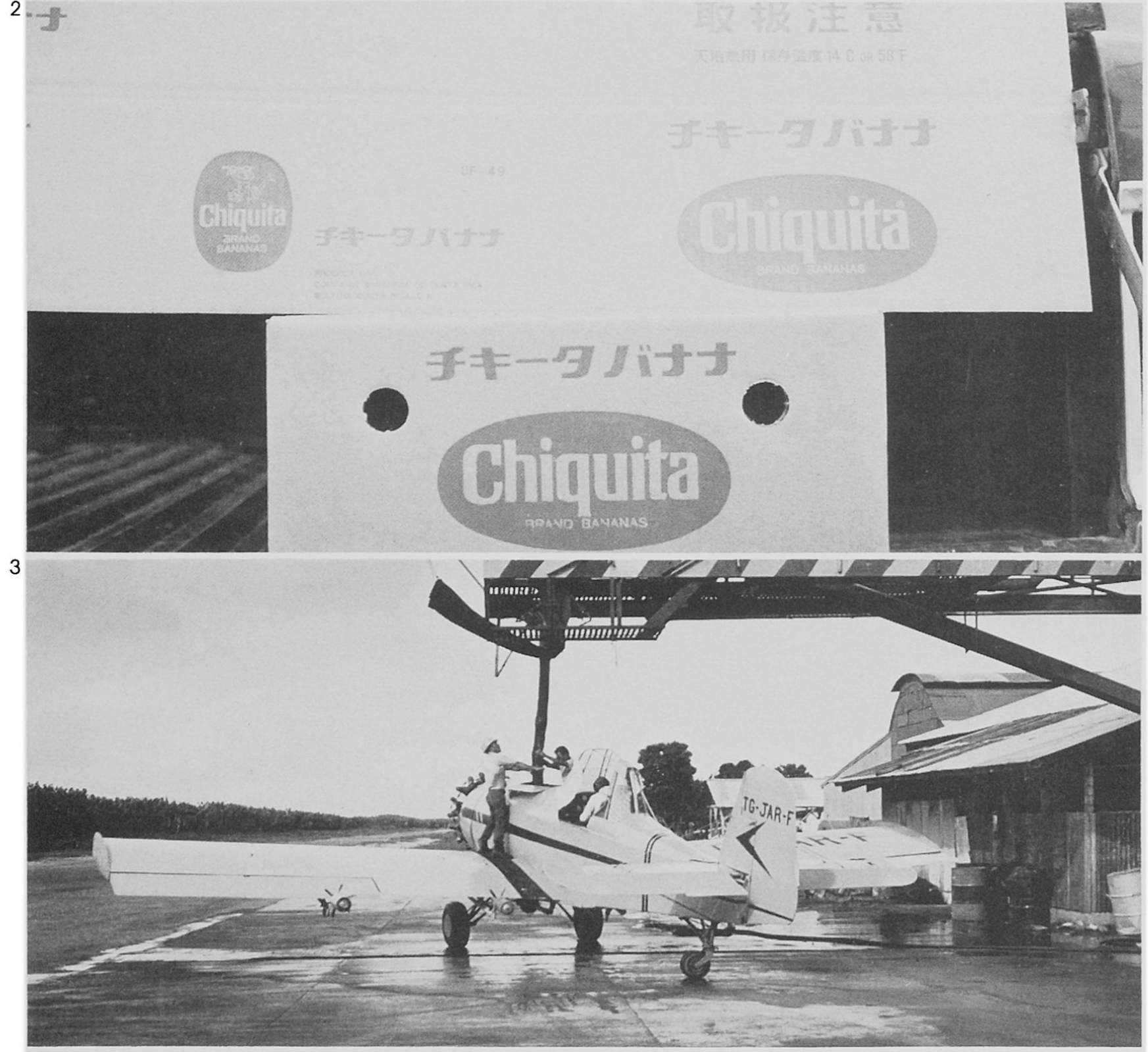

4

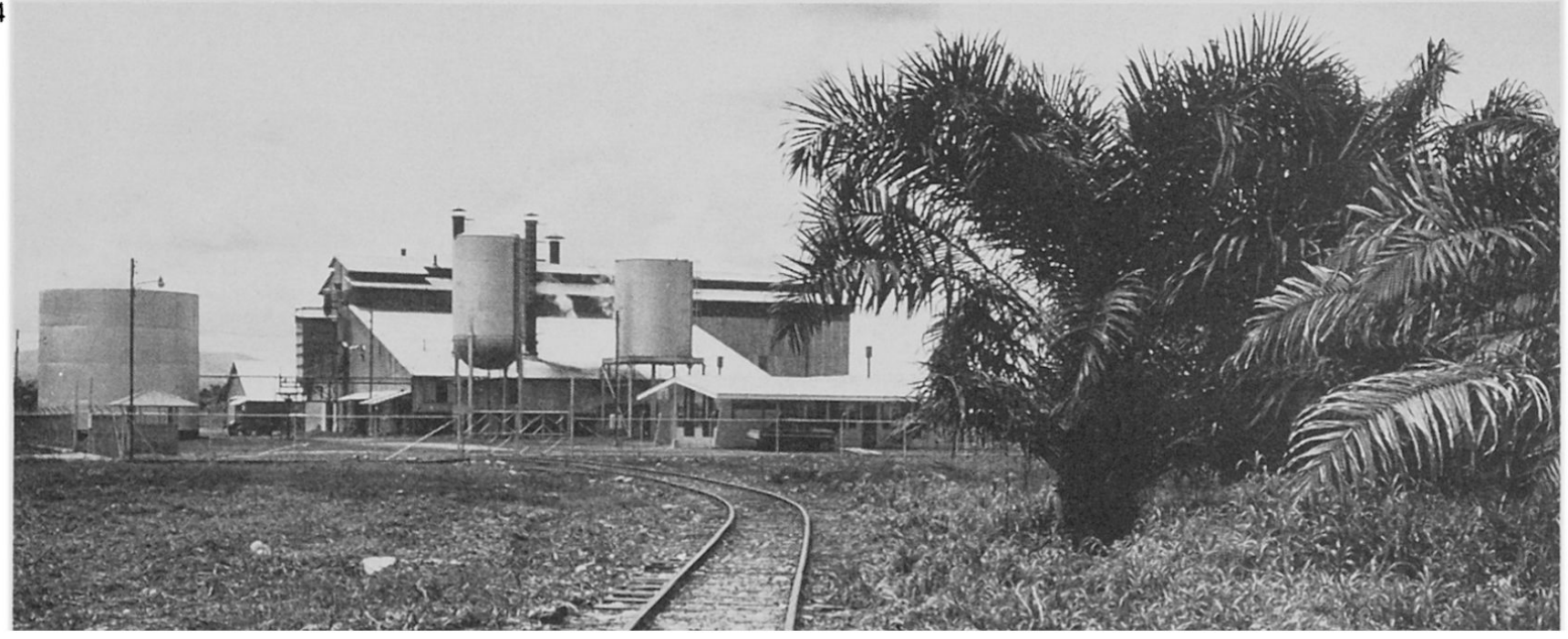


Abb. 1: Ankunft der Bananen«bunches» bei der Verladestation der Farm. Golfito Division, Costa Rica, Juli 1972.

Abb. 2: Heute werden Bananen fast ausschließlich in Kartons verpackt versandt. Ein wichtiger neuer Markt ist Japan. Golfito Division, Costa Rica, Juli 1972.
Abb. 3: Abfüllen eines Flugzeuges mit Spritzmitteln. Bananera, Guatemala, Juli 1972.

Abb. 4: Fabrik zur Gewinnung von Palmöl. Golfito Division, Costa Rica, Juli 1972.
Hafen umgeladen und nach Uebersee transportiert. Diese Kette von Operationen kannte keine Verarbeitung -lediglich stichprobenweise Kontrollen und keine Auffangpuffer. Dies ist heute in jeder Beziehung völlig anders geworden. Jede Pflanzung ist von Kabelbahnen so dicht durchzogen, dass der Transport auf dem Rücken eines Arbeiters nie länger als etwa $30 \mathrm{~m}$ ist. Bei den Kabelbahnen werden die bunches - noch mit den Plastiksäkken bedeckt - wie Würste aufgehängt und durch Verbindungsstücke zu Zügen von bis zu 150 bunches zusammengefügt. Dieses Züge werden entweder durch hängende Motorschlepper oder durch gewöhnliche Traktoren über das System von Kabelstrecken zu der zu jeder Farm gehörenden packing plant gezogen. In dieser Form können bunches ohne weiteres über Nacht in der Auffangstation der packing plant verweilen, wodurch schon ein erster Auffangpuffer gegeben ist, der in der packing plant die Arbeitsaufnahme am frühen Morgen gestattet.

In der packing plant werden zuerst die bunches durch Arbeiterinnen mit scharfen Messern zu sogenannten clusters zerschnitten und die "Stämme" auf Abfall gegeben. An den Schnittstellen erfolgt dabei leicht und rasch eine Infektion, die zur Fäulnis führen kann; die clusters werden deshalb anschliessend einem desinfizierenden Spritzbad unterzogen, bevor sie in Kartons verpackt werden. Die Kartons sind je nach $\mathrm{Be}-$ stimmungsland verschieden - so gibt es beispielsweise japanisch bedruckte Kartons - und auch das Gewicht variiert leicht, beträgt aber in der Regel etwas mehr als $40 \mathrm{~kg}$. Die Kartons werden von einer zentralen Kartonfabrik ( mit Druckerei) flach in die packing plants geliefert und erst dort geformt. Jeder Karton erhält einen Code-Stempelaufdruck, der später en route bei Kontrollen feststellen lässt, auf welcher packing plant und an welchem Arbeitsplatz das Abfüllen geschah. Die Kartons werden direkt in Eisenbahnwagen verladen und im Verladehafen mit Förderbändern - abgesehen von Stichprobenkontrollen - unverändert auf die Dampfer gebracht.

Durch diese neuartige Verpackungsart ergeben sich neben den schon erwähnten noch weitere Vorteile: so wäre es beispielsweise ohne weiteres möglich, die Kartons im Verladehafen in luftkonditionierten Auffangschuppen vorübergehend zu lagern. Ein Nachteil ist, dass schon auf der packing plant die Ware so verpackt werden muss, dass sie auf ein bestimmtes Marktgebiet abgestimmt ist. Dieser Nachteil wird dann noch vergrössert, wenn beispielsweise die Gesetzgebung der USA bestimmte Desinfektionsmittel (Thiabendazol) nicht zulässt. Die Differenzierung bei der packing plant geht heute aber noch weiter. Für bestimmte Absatzgebiete werden Bananen auch einzeln - also nicht in clusters - in standardisierten Grössen in Kartons verpackt. Diese als institutionals bezeichneten Lieferungen sind für Kunden bestimmt, die wie Schulen und Spitäler Bananen rasch und an viele Einzelindividuen verteilenmüssen.

Ausschussbananen gehen heute nur noch teilweise auf Abfallhaufen. In Honduras und in Panama besitzt die UF grosse Fabrikanlagen, wo die Bananen in Wärmekammern in 4 Tagen ausgereift, dann geschält und zu sogenanntem banan mash verkocht werden. Dieses Produkt wird in grosse Fässer verpackt vor allem an die Lebensmittelindustrie (Kindernährmittel, Icecream, Konfiserieartikel usw。) in Uebersee versandt. Erst in den Anfängen - 1972 erstmals in Honduras - steht der Versand in Containern. In diesem Falle werden die Kartons auf der packing plant in Container verladen, die während des Bahntransportes, im Verladehafen, auf dem Schiff usw。 dauernd luftkonditioniert bleiben. Dadurch wird natürlich die Kette der Transportoperationen viel elastischer und der gesamte Arbeitsgang normalisiert. Solange aber nur ein Land die notwendigen Einrichtungen für das Containersystem besitzt, werden die erzielten Vorteile durch eine vermehrte Rigidität des gesamten Transportsystemes kompensiert. Eine bestimmte Kategorie von Dampfern kann nur bestimmte Häfen und keine andern bedienen. Nach dem Zweiten Weltkriege begann die UF damit, auf früherem Bananenland Oelpalmen anzupflanzen。 Heute nimmt diese Pflanze in einzelnen Produktionsgebieten schon ein Drittel der Anbaufläche ein. Im Gegensatz zur Banane spielen hier Krankheiten keine Rolle mit Bezug auf die Ertragsdauer; diese ist vielmehr durch das Höhenwachstum der Oelpalme und die zunehmenden Schwierigkeiten der Ernteoperationen bei etwa 25 Jahren gesetzt. Die Fruchtstände werden im Produktionsgebiet in modernen grossen Fabrikanlagen verarbeitet. Dabei fallen neben dem Palmöl, welches auf dem nationalen Markt abgesetzt wird, als exportables Produkt die Palmkerne an. Ausserdem liefert das Zellulosematerial genügend 
Brennstoff für die gesamte Energieversorgung und zudem ein hochwertiges Aschenmaterial für die Kalidüngung.

Ein beachtenswerter Wandel vollzog sich auch hinsichtlich der Beziehungen zu den Landesregierungen und zu den Arbeiterorganisationen im Rahmen der neuen Sozialgesetze. Allgemein kann gesagt werden, dass durch diese Entwicklungen die Entscheidungsfreiheit der Gesellschaften als Ganzes und gegenüber dem einzelnen Arbeiter in einem Grade eingeschränkt worden sind, die bald die Frage als berechtigt erscheinen lassen, ob eine Fortführung der Produktion wirtschaftlich gerechtfertigt sei. So stimmte beispielsweise die Regierung von Guatemala 1971 der vorgesehenen Uebertragung der Bananenproduktion von UF an Del Monte nicht zu.

Wenn man die Bedeutung der verschiedenen Agrarsektoren in den einzelnen Volkswirtschaften untersucht, ist der Frage der Preisfestsetzung besondere Aufmerksamkeit zu schenken. In den Aussenhandelsstatistiken und den Handels statistiken der FAO erscheinen beispielsweise die beiden für zentralamerikanische Länder besonders wichtigen Ausfuhrprodukte Kaffee und Bananen nach Volumen/Gewicht und Preis. Es handelt sich bei den Preisen jedoch um Angaben, welche nur in beschränktem Umfange miteinander verglichen werden können. Eine Aussage, dass im einen Lande Bananen (aufgrund der ausgewiesenen Exportwerte) wichtiger als Kaffee seien, ist wenig sinnvoll。

Bei Kaffee werden die Preise in einem völlig transparenten Vorgehen für die verschiedenen Kategorien festgelegt und entsprechen dem wahren Handelswert. Das Gut wechselt normalerweise beim Grenzübertritt den Besitzer und die Zahlung erfolgt im angegebenen Umfange tatsächlich von einer Hand an die andere. Alle Gespräche mit Besitzern von Kaffeepflanzungen drehen sich immer um die gegenwärtige oder zukünftige Preisentwicklung. Von ihr hängt der Erfolg, das wirtschaftliche Wohlergehen und die Kreditwürdigkeit des Kaffeesektors und über diesen hinaus eines Grossteiles der Volkswirtschaft ab.

Ganz anders liegen die Dinge im Bananensektor. Der deklarierte Exportpreis kann hier mit vollem Recht als eine fiktive Grösse bezeichnet werden, der nur zollstatistische Bedeutung zukommt. Die Frage nach dem Sinn des Bananenexportpreises wurde bei jeder Gelegenheit auf-, gegriffen; im Gegensatz zum Kaffeesektor muss te aber festgestellt werden, dass es sich dabei um eine Frage handelte, die für Bananenleute völlig uninteressant war und auf welche sie keine Antwort wussten. Tatsächlich findet ja die Handänderung erst. jenseits des Ozeans statt, die Abrechnung erfolgt auf dem Wege über die Zentrale in den USA, Kreditprobleme für Entwicklungsprojekte werden über die gleiche Zentrale gelöst und die cost-benefit Berechnungen der Einzelgesellschaften bleiben vertrauliche Dokumente。

Schliesslich muss noch darauf hingewiesen werden, dass in den letzten zwanzig Jahren das Bananengeschäft auch auf einer höheren Ebene vielgestaltiger wurde. UF wurde zu einer Tochtergesellschaft von United Brands Company. Neben der schon genannten Oelproduktion (Com. pañia Numar Gruppe) gehören zahlreiche weitere Gesellschaften aus dem ganzen weiten Sektor der Lebensmittelindustrie dazu; ausserdem spielt nach wie vor die Tropical Radio Telegraph Company eine wichtige Rolle. Der - Jahresbericht der United Brands Company (6) stellt für 1971 fest: "Wenn auch im vergangenen Jahre 1971 mit Ausnahme der Bananen in den meisten Tätigkeitsbereichen eine Besserung der Geschäftslage festgestellt werden kann, bleibt gesamthaft das Schlussergebnis für die United Brands Company in hohem Masse enttäuschend, vor allem weil sich die Einkünfte aus dem Bananengeschäft drastisch, verminderten. Grund für das rückläufige Geschäftsergebnis war das andauernd gedrückte Preisniveau für Bananen, welches 1971 in praktisch allen Absatzgebieten vorherrschte。"

\section{Bemerkungen}

1) eine heute veraltete Darstellung findet sich bei BOESCH H。: La Tierra del QuetzalZentralamerika heute。1952。

2) Annual Reports der United Fruit Company und der United Brands Company bis 1971 .

3) Zeitschrift TROPICAL AGRICULTURE; vol。 $47 / 3,1970$, p. 233 (L. A。 WALKER)

4) Zeitschrift TROPICAL AGRICULTURE; vol。 47/4, 1970, p. 315 (J.E. EDMUNDS)

5) FAO: Production Yearbook 1971, 1972 。

6) United Brands Company 1971 Annual Report, p. 4,1972 。

Prof. Dr. H. Boesch, Geogr. Inst. Universität Zürich,

Blümlisalpstraße 10, 8006 Zürich 